Review Article

\title{
Contemporary Dentin Bonding Agents-A Review
}

\author{
Nandini Pugal', Praveen rajesh², Dhanavel chakravarthy ${ }^{3}$, Padmaraj S.N ${ }^{4}$, Vijayaraja ${ }^{5}$
}

\begin{abstract}
The purpose of contemporary dentin bonding agent is to know or understand the clinical effectiveness of contemporary resin-based dentin bonding agents primarily focusing on the longevity of restoration. Despite the significant improvements of adhesive systems, the bonded interface remains the weakest area of tooth-colored restorations. The most important reasons for the failure of adhesive restorations with earlier adhesives are the loss of retention and the deficient marginal adaptation. However, the introduction of reliable adhesive restorative materials has subsequently reduced the need for extensive tooth preparation. Dentin bonding has evolved from no-etch to total-etch to self-etch systems. The development of self-etching primer adhesive systems has greatly simplified resin bonding procedures, as a separate etching step is no longer required. There is much interest and activity nowadays with dentin bonding agents. More focus has been laid upon conservative approach of tooth preparation. Therefore, introduction of adhesive restorative materials has reduced the need for an extensive tooth preparation. Modern dental bonding systems come as a "three-step system", where the etchant, primer, and adhesive are applied sequentially; as a "two-step system", where the etchant and the primer are combined for simultaneous application; and as a "one-step system", where all the components should be premixed and applied in a single application (so-called seventh generation of bonding agents So, now the newer generations of bonding agents, self-etch, total etch and their mechanisms and their inner microscopic changes are explained in detail in the forthcoming pages.
\end{abstract}

Key-words: Dentin bonding agent, etch and rinse, wet bonding.

\section{Introduction}

The traditional drill and fill approach is largely a concept of past for the younger generation. Today's operative dentistry primarily involves "minimally invasive" care because the dental profession now recognizes that an artifact is of less biological value than the original healthy tissue. The adhesive materials have revolutionized dentistry by opening up conservative cavity preparation options. Also, adhesive techniques promote "maintenance and repair" instead of changing the entire restorations which has further boosted its use in varied applications of everyday clinical practice. ${ }^{(1,2)}$

The primary factor in the premature failure of moderate to large composite restorations is secondary caries at the margins, particularly the gingival margin of the restorations due to unreliable dentin bonding. ${ }^{(3,4)}$ These resin based materials tend to have a major weak link at the resin-dentin interface. Although bonding to enamel has been quiet predictable, bonding to dentin poses a million-dollar challenge till date. Modern adhesive system is superior to their predecessors especially in terms of retention that is no longer a major cause of premature clinical failure. ${ }^{(5)}$ Though the current adhesive systems have tremendously improved the complex dentin bonding there are still changes that are needed to be addressed.

Over the last 40 years, there seemed to be an expansion of knowledge in this area major advances have been made in both adhesive monomer formulations and in pre-treatment of dentin to improve resin penetration inside the tissue matrix. It is hoped that this review will stimulate critical thinking in this area and encourage new research into problems yet unsolved. ${ }^{(7)}$.

The concept of adhesive has been essentially the most noteworthy development in ever progressing sciences, over the past three decades bonding of resin-based composite filing has been revolutionized by advances in dental bonding technology. ${ }^{(8)}$

There has been a noteworthy alteration in the principles of cavity preparation design, from the age old principles of "extension for prevention" defined by G.V. Black to a more carious lesion-centered method. This lesion-centered method is promising through the innovations in adhesive restorative materials and through the introduction of computer-assisted ways and means of caries detection, an enhanced understanding of the role of magnification, digital radiography and caries 
risk assessment of the patient to permit for developed conservative caries management. ${ }^{(10)}$

\section{Composition Of Dentin Bonding Agents}

\section{Etchants:}

Etchants are strong acids which are used to remove smear layers and open tubules, increase retention of resin sealant and promote mechanical retention. They dissolve minerals and allow the formation of micromechanical interlocking in enamel and dentin $30-50 \%$ of phosphoric acid can be used but exactly $37 \%$ of phosphoric acid is preferred for etching to produce consistent etching pattern which do not damage the pulp. If the concentration is at $50 \%$ or greater than $50 \%$ then it results in the deposition of adherent layer of mono-calcium phosphate monohydrate on the etched surface which inhibits further dissolution. Etchant is supplied as an aqueous gel to allow placement over a specific area. Composition of these gels is colloidal silica or polymer beads. Other materials used as etchants are maleic acid, tartaric acid, citric acid, EDTA, acidic monomers, polyacrylic acid, hydrochloric acid, nitric acid and hydrofluoric acid.

\section{Patterns of etching:}

\begin{tabular}{|l|l|}
\hline Type 1 & $\begin{array}{l}\text { Most common etching pattern. This } \\
\text { involves removal of enamel prism } \\
\text { cores with prism peripheries remaining } \\
\text { leaving intact }\end{array}$ \\
\hline Type 2 & $\begin{array}{l}\text { Here peripheries are removed leaving } \\
\text { the cores intact. }\end{array}$ \\
\hline Type 3 & $\begin{array}{l}\text { This is associated with the presence of } \\
\text { prism less enamel }\end{array}$ \\
\hline
\end{tabular}

\section{Primers:}

Primers are solutions containing hydrophilic monomers that get dissolved in a solvent like acetone, ethanol or water. Such monomers exhibit hydrophilic properties through

HEMA- Phosphate, phenyl $\mathrm{p}\{(2-$ methacryloyloxyethy) phenyl hydrogen phosphate)\}-Phosphate, carboxylic acid, alcohol.

10-MDP (metharyloyloxydecyl dihydrogen phosphate)

4-meta (4-methalacryloyloxyethyl tri mellitic acid)
4-META(4-methacryloyloxyethyl1 tri mellitic anhydride)

MAC-10(11methacryloyloxy-1,1-undecanedicarboxylic acid

\section{Solvent:}

The addition of solvents to resin is indispensable to the composition of adhesives that needs to be bonded to the dentine. Primers/adhesives have low viscosity due to dissolution of monomers in a solvent that improves their diffusion ability in the micro retentive tooth surface. Most commonly used solvents are water, ethanol and acetone. Water ionizes acid monomers and re-expands the collagen network which had already collapsed. Ethanol is used instead of water as co-solvent. Water-alcohol combination mixtures start the formation of hydrogen bonds between water and ethanol molecules which leads to better evaporation of water ethanol aggregate than plain water. Acetone consists of high vapor pressure. The advantage is that the pressure is four times higher than ethanol. It is highly volatile which has a less shelf life by rapid evaporation. Both ethanol and acetone can remove water easily as they have better miscibility with hydrophobic monomers. So they are called "water chasers".

The main purpose of adhesive is to fill inter-fibrillar spaces of the collagen network that created a hybrid layer and resin tags for micromechanical retention.

Adhesive is composed of

BIS-GMA-Bisphenol Glycidyl Methacrylate

TEGDMA-Tri Ethyl Glycol Dimethacrylate

UDMA -Urethane Dimethacrylate

and small amounts of HEMA -2 Hydoxy Ethyl Methacrylate

\section{Initiators:}

The main purpose of the initiator is polymerization. Polymerization can be initiated by photo initiator system which consists of camphorquonine a photo sensitizer and an initiator -tertiary amine through a self-cure system a chemical initiator like benzoyl peroxide or through dual cure initiator system, fillers: these fillers are added to the resin to reinforce the adhesive it gives a strengthening effect other reason is to modify the viscosity of the adhesive to a thicker to pastier consistency. 


\section{Mechanism of action:}

Many trials have been made to achieve this type of bonding, but till now there are very few evidence that such bonds form. The reason simply is that the tooth surface is poorly standardized regarding chemical composition and that the bonds are not stable when other ions, diffuse along the interface and compete with the different bond sites.

\section{Bonding to Hydroxyapatite:}

The formula M-R-X) shows the chemical bonding in dentistry.

$\mathrm{M}$ - Stands for the methyl methacrylate group including the -CO-O- bond).

$\mathrm{R}$ - stands for a spacer consisting of a hydrocarbon chain,

$\mathrm{X}$ - is the group capable of bonding to calcium present on the tooth surface.

\section{HYBRID LAYER:}

The distinct zone between the bulk adhesive and nondemineralized dentin (i.e.,) consisting of $50 \%$ collagen matrix and $50 \%$ resin is termed as hybrid layer. Hybrid layer is formed when an adhesive resin penetrates a de-mineralized or acid etched dentin surface infiltrates the visible collagen fibrils (fig: 1 ). The hybrid layer is very tough when it is correctly formed and shows good micromechanical retention for resin ${ }^{(13)}$ Infiltration of demineralized collagen fibers with resin and formation of hybrid layer results in Successful bonding.

Development of hybrid layer is a vital part of dentin bonding. The superiority of the hybrid layer that is formed decides the strength of resin-dentin interface. In2009, Ana Paula Martin specified that micromechanical behavior of hybrid layer along with voids should lower bond strength of self-etching adhesives then in 2011 they concluded that bonding agent with simplified application procedure were less successful compared to conventional totaletch adhesives. ${ }^{(14)}$ Traditional enamel bonding theory postulates that resin tags mechanically lock into undercuts in the etched enamel.

The hybrid concept proposes that bonding stability will increase if the resin tags are supplemented by a thin zone of hybrid layer (resin-reinforced tissue). The hybrid layer forms an acid resistant envelope that seals the dentin, preventing from hypersensitivity and secondary caries.
Three characteristics are necessary for the formation of hybrid layers

1. The aggressive acid expose collagen below the hybrid layer and leaves a zone of weak dentin that leads to long term degradation

2. The bonding resin must include monomers with both hydrophilic and hydrophobic groups that can penetrate the dentin and combine with that.

3. The catalyst present here should allow polymerization process in the presence of oxygen and water.

There are three different zones in hybrid layer:

\section{Top zone:}

It consists of loosely arranged collagen fibrils with inter-fibrillary spaces filled with the adhesive resin

\section{Mid-zone:}

This consists of collagen fibrils infiltrated with resin with residual hydroxyapatite crystals between collagen

\section{Bottom zone:}

It shows an abrupt transition to the underlying unaltered dentin with partially de-mineralized dentin containing hydroxyapatite crystals enveloped by adhesive resin. The resin monomers are not able to fully infiltrate the de-mineralized dentin, only a part of de-mineralized dentin remains susceptible to hydrolytic degradation. The remaining water gets entrapped in the hybrid layer and deteriorates the bond strength. The hydrophilic monomers act as a semi permeable membrane which allows the movement of water, classically known as "water tree" phenomenon. This exposes both resin and exposed collagen fibers to degradation by hydrolysis. ${ }^{(17)}$

Hybrid layer is formed by the penetration of monomer in dentin and its polymerization in situ which is essential for a good bonding to dentin.

The other one is the reverse hybrid layer in which the hybrid layer is surrounded by more inorganic material. The hybrid layer degrades coincidently with decreasing bond strength which contributes to the ageing of dentin 
bond integrity. This is caused by the activation of MMP's which denature the collagen in the hybrid layer. ${ }^{(1)}$

As the smear layer is a porous substrate, the expectation is that thick smear layers could work as water reservoir and accelerate the degradation of the bonds. The hybridized smear layer has as much attraction toward water. Various advancements in the properties of adhesive systems have been developed to reduce micro leakage by strengthening the adhesion between the tooth surface and the restoration, such as increasing the acid potential of the etchant, addition of Nano fillers, and change in resin composition.

\section{Total Etch Technique}

Fusayama advocated etching enamel and dentin simultaneous. This was a very opposing concept in the United States and Europe. acid-etching dentin produces pulpal death. A review of the literature indicated that the pulpal reactions which occurred after acid etching were largely because of inadequate sealing of etched cavities, and were the consequence of bacterial leakage.

Few years ago they found, reduction in etching time and improvements in bonding formulations and techniques have eliminated the acid-etching controversy. Bonding was a 3 -step process:

1. Total etch and rinse

2. Priming and evaporation of solvent and

3. Application of the adhesive then light curing.

1. Acid- it uses 35-37\% phosphoric acid which removes the smear layer and de-mineralizes the most superficial hydroxyapatite crystals, thus exposing both intertubular and peritubular collagen.

2. Primer- Includes bi-functional molecules (hydrophilic and hydrophobic).

3. Resin- Includes monomers that are mostly hydrophobic; such as Bis-GMA. The resin monomers permeate the water filled spaces between adjacent dentin collagen fibers that used to be occupied by hydroxyapatite crystals. Several dental product manufacturers brought fourthgeneration bonding systems that etch dentin with phosphoric acids and some other acids. Examples include All- Bond 2, Amalgam bond,
Clearf l Liner Bond OptiBond (Kerr), ProBond and Scotchbond Multi-Purpose Plus (3M). Many investigators have reported shear bond strengths for these materials that approach or exceed the typical enamel bond strength of $20 \mathrm{MPa}$. In addition, micro leakages studies indicate that they provide a better marginal seal than earlier generations of adhesives.

A separate acid-etching step is still needed in "totaletch" wet-bonding techniques. This is done by application of two layers of bonding agent to wet dentin. The first layer is the same purpose as did the original primer. It removes much of the residual water and starts into penetrate adhesive monomer into acid etched dentin. If the acid-etched dentin surface is too wet, the change of phase occurs. Generally, when the second layer of bonding agent is applied, fresh monomers and their solvent re-dissolve the resin globules, leaving a more homogeneous film.

\section{Ethanol Wet Bonding:}

The Ethanol wet-bonding concept was emerged from the tissue embedding techniques in which hydrated organic tissues are chemically dehydrated with ethanol for a few hours and then they will be embedded into epoxy resin. Due to the presence of water in the current wet-bonding techniques, there is a risk for phase separation of the hydrophobic Bis-GMA monomer from the hydrophilic resin monomers, which has limited water solubility. To avoid these problems, the wet-bonding technique involved the use of hydrophilic monomers, such as Hydroxyethyl methacrylate (HEMA). These hydrophilic monomers absorb moisture after polymerization, which causes plasticization of the resin polymer chains and thereby decreases the resin-dentine bond strength. Then the "ethanol-wet bonding (EWB)" concept was developed to replace water with ethanol to support the de-mineralized dentine collagen fibrils. ${ }^{(26)}$

This ethanol dehydration process is known as "full chemical dehydration protocol" given by Sadek et al. and takes more time and it is too complex to perform properly in a clinic daily.

When dentin is treated with an acidic conditioner is rinsed with water, the solubilized calcium and phosphate ions and reaction products should be extracted from the spaces between the collagen fibrils. When "wet bonding" techniques applied, the collagen fibril network is suspended in water, blocks the penetration of resin half way. Unlike the conventional water-wet-bonding 
technique, this method uses ethanol instead of water to saturate and to prevent the collapse of de-mineralized dentin matrices before to resin application. Water is replaced from inter-fibrillar spaces with ethanol during saturation of de-mineralized dentin matrices with ethanol before the application of the adhesive.

The rationale behind this is that miscibility of adhesive resin monomers in the ethanol-saturated dentin matrices is better than those in the water-saturated dentin matrices. So ethanol is used as a saturation solvent for de-mineralized dentin matrices which allows intimate encapsulations of collagen fibrils with adhesive resin monomers. Therefore, ethanol-wet bonding might destroy enzymatic degradation of collagen fibrils and improve durability of resin dentin bonds ${ }^{(27)}$

The advantage of Ethanol-wet bonding technique is that it can successfully persuade less hydrophilic monomers into the dentine matrix, creating a more hydrophobic hybrid layer that could absorb less water. Adhesives should be applied on time to ethanol-wet dentine to permit better infiltration of the adhesive monomers. The timeliness results in the avoidance of the collapse of the collagen network caused by the quick Evaporation of ethanol. ${ }^{(28)}$.

\section{Self Etch Adhesive}

Self-etch adhesives are developed for reduction in application steps and for hemical interaction with hydroxyapatite-coated collagen fibers. ${ }^{(32)}$ Instead, bonding to dentin with additional phosphoric acid etching still remains controversial.

The idea of self-etching approach was created approximately 20 years ago, though, the first and second generations of bonding agents can be considered self-etch materials since no acid etching/rinsing or conditioning step were used. Self-etch adhesive systems also comprise HEMA monomer because most of the acidic monomers are low water-soluble and to rise the wettability of dentin surface. Bi- or multi-functional monomers are added to provide strength to the cross-linking formed from monomeric matrix ${ }^{(5)}$. Because self-etch adhesive systems do not need a separate acid conditioning step and moist post-rinse control, they are considered simplified adhesive materials. ${ }^{(32)}$ self-etch approach may have the best future perspective. They are categorized as Ultra mild self-etc., Mild self-etch, Intermediate self-etch, Strong self-etch

\section{GIC Based Adhesive}

Glass-ionomers continue as the only materials that are self-adhesive to tooth tissue without any surface pretreatment. Pre-treatment with a feeble poly- alkenoicacid conditioner significantly improves bonding. Glassionomer approach can be achieved following a one- or two-step application procedure The extra conditioning step becomes more important, when coarse cutting diamonds are used and, so, thicker and more compact smear layers are produced. Poly-alkenoic-acid conditioner is applied for 10-to-20 seconds and gently rinsed off, followed by gently air-drying without dehydrating the surface. The increase in bonding efficiency must be partially attributed by

- "Cleaning" effect, by which cutting debris is removed, a partial "demineralization" effect, by which the surface area is enlarged

- Micro porosities for micromechanical meshing or hybridization are visible

- Chemical contact of poly-alkenoic acid with residual hydroxyapatite ${ }^{(31)}$

Glass ionomer cements (GIC) are often used as a lining or base material under resin composite restorations as a dentin replacement to seal the dentin with a material demonstrated to form a dependable bond. There is presently very little research data on the topic of bonding of GIC and resin-based adhesives, even if this technique is widely recommended for the restoration of large proximal cavities. Micromechanical bond is also significant to bond to a conventional GIC. In the case of resin-modified GICs, there is also a chemical bond between the resin constituent of the resin-modified GIC and resin of the enamel/dentin adhesive.

\section{Conclusion}

Improvements have been made for aesthetic appeal, ease of use and reduction of technique sensitivity. The innovation of dentin bonding agents has solved many issues like the application procedure and bonding and thus making the procedure minimally time conducive. As we enter the new millennium it is important to examine the past keeping abreast of the fast rapidly spreading progresses in the drill of adhesive dentistry with the hottest trend. 


\section{References}

1. Van Meerbeek B, De Munck J, Yoshida Y, Inoue S, Vargas M, Vijay P, Van Landuyt K, Lambrechts P VGB. Adhesion to enamel and dentin: current status and future challenges. Oper Dent. 2003;28:215-35.

2. Van Niuwenhuysen JP, D’Hoore W, Carvalho J, Qvist V. Longterm evaluation of extensive restorations in permanent teeth. $\mathrm{J}$ Dent 2003; 31: 395-405 (Pub Med: 12878022).

3. Hunter AR, Treasue ET, Hunter AJ. Increases in cavity volume associated with the removal of class 2 amalgam and composite restorations. Operat Dent 1995; 20:2-6

4. Kleverlaan CJ, Feilzer AJ. Polymerization shrinkage and contraction stress of dental resin composites. Dent Mater 2005;21:1150-1157.

5. Abedin F, Ye Q, Parthasarathy R, Misra a., Spencer P. Polymerization Behavior of Hydrophilic-Rich Phase of Dentin Adhesive. J Dent Res [Internet]. 2015;94(3):500-7. Available from: http://jdr. sagepub. com/ cgi/doi/ 10.1177/ 0022034514565646YiuC

6. An overview of solvents in resin-dentin bonding. 2014;(January)

7. On AF, Disease S. Current Concepts. 2009;2(2):1-8

8. Gupta S, Biswal SS, Kaushik SV. Review Article Dentin Bonding Agents : An Overview. :82-4.

9. Communication S. Dentin Bonding Agents I : Complete Classification - A Review. 2011;2(December):367-70.

10. Strassler HE, Mann M. Dental Adhesives for Direct Placement Composite Restorations : An Update.

11. Journal of esthetic dentistry and restorative dentistryvol 27,n0.6.331- 334.2015doi.10.1111/jerd.12185 by Jorge perdigao,edwardnj.swift.j.r.dmd,ms

12. Phillips ciences of dental materials Anusavice/shen/rawls/Elsevier

13. Agee K, Chiba A, Tagami J. HHS Public Access. 2016;28(6):70621

14. Roberson TM. EDITORS.-sturdevants operative dentistry book

15. Inoue G, Nikaido T, Foxton RM, Tagami J. The acid-base resistant zone in three dentin bonding systems. 2009;28(6):717-21

16. Di Francescantonio, M., de Oliveira, M. T., Shinohara, M. S., Ambrosano, G. M. B., \& Giannini, M. (2007). Bond strength evaluation of self-etch and total-etch adhesive systems on intact and ground human enamel. Brazilian Journal of Oral Sciences, 6(23), 1462-1466

17. Helvey $\mathrm{G}$ a. Creating super dentin: using flowable composites as luting agents to help prevent secondary caries. Compend Contin Educ Dent [Internet]. 2013;34(4):288-300. Available from: http:// www. ncbi.nlm. nih.gov/ pubmed/23895566

18. Nikaido T, Inoue G, Takagaki T, Waidyasekera K, Iida Y, Shinohara MS, et al. New strategy to create "Super Dentin" using adhesive technology: Reinforcement of adhesive-dentin interface and protection of tooth structures. Jpn Dent Sci Rev. 2011;47(1):31-42.

19. Aggarwal V, Singla M, Sharma R, Miglani S, Bhasin SS. Effects of simplified ethanol-wet bonding technique on immediate bond strength with normal versus caries-affected dentin. J Conserv Dent [Internet]. 2016;19(5):419-23. Available from: http://www.ncbi. nlm.nih.gov/ pubmed $/ 27656059$

20. Van Landuyt K, De Munck J, Coutinho E, Peumans M, Lambrechts P, Van Meerbeek B. Bonding to dentin: Smear layer and the process of hybridization. Dental Hard Tissues and Bonding: Interfacial Phenomena and Related Properties. 2005. 89-122 p.

21. Tjäderhane L, Nascimento FD, Breschi L, Mazzoni A, Tersariol ILS, Geraldeli S, et al. Optimizing dentin bond durability: Control of collagen degradation by matrix metalloproteinases and cysteine cathepsins. Dent Mater [Internet]. 2013;29(1):116-35.

22. Liu Y, Tjäderhane L, Breschi L, Mazzoni A, Li N, Mao J, et al. Limitations in bonding to dentin and experimental strategies to prevent bond degradation. J Dent Res. 2011;90(8):953-68.

23. The Evolution of Dentin Bonding | Dentistry Today [Internet]. Available from: http://dentistrytoday.com/materials/1483

24. Parmar G. Review article : Hybrid layer : Foundation of Dental bonding. 2014;(October):46-50.

25. Swift EJ. Dentin/enamel adhesives: review of the literature. Pediatr Dent. 2002;24(5):456-61.

26. Ekambaram M, Kar C, Yiu Y, Pekka J, Wen J, Chang W, et al. ScienceDirect Effect of chlorhexidine and ethanol-wet bonding with a hydrophobic adhesive to intraradicular dentine. J Dent [Internet]. 2014;1-11. Available from: http://dx.doi.org/10.1016/j. jdent.2014.02.006

27. Ekambaram M, Kar C, Yiu Y, Pekka J, Martyn N, Russell F. ScienceDirect Adjunctive application of chlorhexidine and ethanol-wet bonding on durability of bonds to sound and cariesaffected dentine. J Dent [Internet]. 2014;42(6):709-19. Available from: http://dx. doi.org/ 10.1016/ j.jdent.2014.04.001

28. Effect of simplified ethanol-wet bonding on microtensile bond strengths of dentin 2adhesive agents with different solvents. 2016;(October).

29. Pei D, Huang X, Huang C, Wang Y. Ethanol-wet bonding may improve root dentine bonding performance of hydrophobic adhesive. J Dent [Internet]. 2012;40(5):433-41. Available from: http://dx.doi. org/10. 1016/j. jdent.2012.02.005

30. Article R, Pathology M. From dry bonding to water-wet bonding to ethanol-wet bonding. A review of the interactions between dentin matrix and solvated resins using a macromodel of the hybrid layer. 2007 Swift j. Review. 1998;

31. Kenshima S, Francci C, Reis A, Dourado A, Eloy L, Filho R. Conditioning effect on dentin, resin tags and hybrid layer of different acidity self-etch adhesives applied to thick and thin smear layer. 2006;34:775-83. 
32. Giannini M, Makishi P, Almeida Ayres AP, Moreira Vermelho P, Marin Fronza B, Nikaido T, et al. S e $1 \mathrm{f}-\mathrm{E}$ t $\mathrm{c}$ h Ad h e s i v e S y s t e m s : A Literature Review. Braz Dent J. 2015;26(1):3-10.

33. Zhang Y, Burrow M, Palamara J, Thomas C. Bonding to Glass Ionomer Cements Using Resin-based Adhesives. Oper Dent. 2011;36:618-25.

\section{Address of Correspondence}

Dr. Nandini pugal,

Final Year Postgraduate

Department of Conservative dentistry and endodontics

Indira Gandhi institute of dental sciences.

Email id: nandini26588@gmail.com

Phone no: 9952184648
34. Carious Dentin Treatment For Glass Ionomer Cement Adhesion : A Comparative Study Carious Dentin Treatment For Glass Ionomer Cement Adhesion : A Comparative Study. 2010;1-11.

\section{Authors}

${ }^{1}$ Final year postgraduate, department of conservative dentistry and endodontics, Indira Gandhi institute of dental sciences

${ }^{2,4}$ Reader, Department of Conservative dentistry and endodontics, Indira Gandhi institute of dental sciences

${ }^{3}$ Professor and Head, Department of Conservative dentistry and endodontics, Indira Gandhi institute of dental sciences

${ }^{5}$ Senior lecturer, department of conservative dentistry and endodontics, Indira Gandhi institute of dental sciences

How to cite this article : Nandini Pugal, Praveen rajesh, Dhanavel chakravarthy, Padmaraj S.N, Vijayaraja. Contemporary Dentin Bonding AgentsA Review. Journal of Scientific Dentistry 2018;8(2):13-9

Source of support : Nil, Conflicts of Interest : None declared 